RESPIRATORY INFECTION

\title{
Aetiological role of viral and bacterial infections in acute adult lower respiratory tract infection (LRTI) in primary care
}

\author{
D D Creer, J P Dilworth, S H Gillespie, A R Johnston, S L Johnston, C Ling, S Patel, \\ G Sanderson, P G Wallace, T D McHugh
}

See end of article for authors' affiliations

Correspondence to: Dr J P Dilworth

Department of Thoracic Medicine, Royal Free Hospital, London NW3 2QG, UK; paul.dilworth@ royalfree.nhs.uk

Received 6 May 2004 Accepted

21 September 2005

Published Online First

14 October 2005

Background: Lower respiratory tract infections (LRTI) are a common reason for consulting general practitioners (GPs). In most cases the aetiology is unknown, yet most result in an antibiotic prescription. The aetiology of LRTI was investigated in a prospective controlled study.

Methods: Eighty adults presenting to GPs with acute LRTI were recruited together with 49 controls over 12 months. Throat swabs, nasal aspirates (patients and controls), and sputum (patients) were obtained and polymerase chain reaction (PCR) and reverse transcriptase polymerase chain reaction (RT-PCR) assays were used to detect Streptococcus pneumoniae, Mycoplasma pneumoniae, Chlamydia pneumoniae, Legionella pneumophila, influenza viruses ( $\mathrm{AH} 1, \mathrm{AH} 3$ and $\mathrm{B})$, parainfluenza viruses 1-3, coronaviruses, respiratory syncytial virus, adenoviruses, rhinoviruses, and enteroviruses. Standard sputum bacteriology was also performed. Outcome was recorded at a follow up visit.

Results: Potential pathogens were identified in 55 patients with LRTI (69\%) and seven controls (14\%; $p<0.0001$ ). The identification rate was $63 \%$ (viruses) and $26 \%$ (bacteria) for patients and $12 \%$ $(p<0.0001)$ and $6 \%(p=0.013)$, respectively, for controls. The most common organisms identified in the patients were rhinoviruses $(33 \%)$, influenza viruses $(24 \%)$, and Streptococcus pneumoniae (19\%) compared with $2 \%(p<0.001), 6 \%(p=0.013)$, and $4 \%(p=0.034)$, respectively, in controls. Multiple pathogens were identified in 18 of the 80 LRTI patients $(22.5 \%)$ and in two of the 49 controls (4\%; $p=0.011$ ). Atypical organisms were rarely identified. Cases with bacterial aetiology were clinically indistinguishable from those with viral aetiology.

Conclusion: Patients presenting to GPs with acute adult LRTI predominantly have a viral illness which is most commonly caused by rhinoviruses and influenza viruses.

$\mathrm{R}$ espiratory tract infections are the most common reason for primary care consultations. ${ }^{1}$ One third of all respiratory tract infections are lower respiratory tract infections (LRTI) ${ }^{2}$ with an incidence of 44-50 per $1000 .^{34}$ Most LRTI in primary care in Europe including the UK are treated with antibiotics, ${ }^{2}$ despite the limited evidence of clinical benefit and the fact that overuse of antibiotics is associated with an increasing rate of antibiotic resistance. ${ }^{5}$

The aetiology of acute LRTI presenting to primary care physicians is also poorly established due to inadequate patient definition and limited pathogen detection (16$55 \%)^{3}{ }^{4-8}$ resulting from a combination of inadequate clinical sampling and pathogen detection methodology, particularly for respiratory viruses. For example, a study using serology for respiratory viruses and atypical bacteria found evidence of infection in only $16 \%$ of adults with acute bronchitis. ${ }^{8}$ Yields of $29 \%$ and $40 \%$ were achieved when viral culture of nasopharyngeal washings and sputum culture for bacteria was added. ${ }^{67} \mathrm{~A}$ diagnostic yield of $44 \%$ was achieved, but required a range of samples including induced sputum, urine, saliva, throat swab and blood for serology to identify pathogens. ${ }^{4}$

Thus, little is known about the aetiology of ambulatory patients with mild or moderate LRTI without evidence of pneumonia. Moreover, none of the previous studies investigating the aetiology of LRTI in primary care has included organism detection in a control population. A comparison of the detection rates of potential viral and bacterial pathogens between patients and matched controls is essential in attributing a pathological role for the organisms detected. We present the results of a 12 month observational study of the aetiology of LRTI using both culture and nucleic acid amplification techniques for the detection of potential pathogens.

\section{METHODS \\ Subjects}

Adult patients ( $\geqslant 18$ years) were recruited from two general practices with a multi-ethnic patient population of 15000 from social classes I-V. All patients were surgery attendees; no recruitment was undertaken out of hours or on home visits. Acute LRTI were defined as a new or worsening cough and at least one other lower respiratory tract symptom for which there was no other explanation, present for $\leqslant 21$ days. ${ }^{3}$ Patients were excluded if they had underlying chronic suppurative lung disease (defined as bronchiectasis, lung abscess or empyema), tuberculosis, immunodeficiency, or previous study participation ( 3 weeks). Antibiotic prescription, investigation, and follow up were at the discretion of the GP. Age, sex, and season matched controls were recruited from general practice patients attending for nonrespiratory and non-infective illnesses and other healthy volunteers with no history of respiratory tract symptoms for 2 months prior to recruitment using the same exclusion

Abbreviations: LRTI, lower respiratory tract infections; RSV, respiratory syncytial virus; RT-PCR, reverse transcriptase polymerase chain reaction 
criteria as patients. Recruitment took place between May 2000 and April 2001.

The study was approved by the Royal Free NHS trust local ethics committee and written consent was obtained from all patients and controls before inclusion into the study.

\section{Study design}

All patients were seen by the study research fellow (DDC) at the initial GP consultation and at a planned visit 4 weeks later. Demographic and clinical data (duration and nature of symptoms, past medical history, pulse rate, respiratory rate, and temperature) and details of the patient's management (antibiotic treatment) were recorded.

\section{Specimen collection and processing}

Throat swabs, nasal aspirates, and blood for C-reactive protein (CRP, normal range $<5 \mathrm{mg} / \mathrm{dl}$ ) were collected from patients and controls and sputum was collected from patients when possible at the first visit. In those patients who were prescribed antibiotics, specimens were obtained before the antibiotics were taken.

\section{Samples analysed by viral and atypical bacterial reverse transcriptase polymerase chain reaction (RT-PCR)}

Nasal aspirates were collected, mixed with $4 \mathrm{ml}$ of viral transport medium (VTM), aliquoted and stored at $-70^{\circ} \mathrm{C}$ within 1 hour of sampling. ${ }^{9}$ When rhinorrhoea was absent or minimal it was induced using a single $100 \mu \mathrm{l}$ nasal spray to each nostril of sterile $8 \mathrm{mg} / \mathrm{ml}$ histamine (Nova Laboratories Ltd, Leicester, UK). ${ }^{9}$ A sputum plug was mixed in $4 \mathrm{ml} \mathrm{VTM,}$ aliquoted into four sterile RNase free microtubes (Bioquote Ltd) and stored at $-70^{\circ} \mathrm{C}$ within 1 hour of sampling.

\section{Samples analysed by bacterial PCR and by standard} sputum culture

Sputum was processed on the day of collection and standard Gram's staining and bacterial culture performed. Heat killed sputum aliquots were stored for PCR at $-70^{\circ} \mathrm{C}$. Throat swabs were transported to the laboratory within 24 hours of collection, stored at $4^{\circ} \mathrm{C}$ for up to 48 hours, and then stored at $-70^{\circ} \mathrm{C}$.

\section{Sample processing for PCR \\ DNA extraction for PCR}

Throat swabs were expressed in $1 \mathrm{ml}$ DNase free sterile water. For DNA extraction either $250 \mu \mathrm{l}$ of throat swab diluent or $250 \mu \mathrm{l}$ sputum aliquots were mixed with an equal volume of $10 \%$ Chelex (Sigma, UK) and the supernatant removed after centrifugation and used as the sample template for PCR. DNA was extracted from positive controls (Streptococcus pneumoniae, Staphylococcus aureus, Haemophilus influenzae, Mycoplasma pneumonaie, Legionella pneumophila, Mycobacterium tuberculosis, Escherichia coli, Pseudomonas aeruginosa, group C streptococcus, viridans type streptococci, coagulase negative staphylococcus, and Candida species) by proteinase $\mathrm{K}$ and $10 \%$ sarkosyl digestion followed by a standard phenol-chloroform extraction method. The positive control for Chlamydia pneumoniae was a plasmid containing the $C$ pneumoniae outer membrane protein gene.

\section{RNA extraction for RT-PCR}

RNA extraction from $140 \mu \mathrm{l}$ sputum and nasal aspirate aliquots and positive controls (influenza viruses AH1, AH3 and B, parainfluenza viruses 1,2 and 3, rhinovirus 16, coronaviruses 229E and OC43, RSV, adenovirus, M pneumoniae and C pneumoniae) was performed using QIAamp viral RNA mini spin columns following the manufacturer's instructions (QIAGEN Ltd, West Sussex, UK).

\section{Reverse transcription}

RNA was reverse transcribed using both random hexamers (Promega, Southampton, UK) and a specific picornavirus (rhinovirus and enterovirus) primer OL27 ${ }^{\circ}$ (Oswel, Southampton, UK) to produce cDNA representative of all RNA species in the original clinical sample and picornavirus specific cDNA, respectively.

\section{PCR protocols}

The RT-PCR panel was used to identify $M$ pneumoniae, ${ }^{10} \mathrm{C}$ pneumoniae, ${ }^{11}$ influenza viruses $\mathrm{AH}, \mathrm{AH} 3$ and $\mathrm{B}^{12}$ parainfluenza viruses $1-3,{ }^{13}$ coronaviruses $229 \mathrm{E}$ and OC43, ${ }^{14}$ respiratory syncytial virus (RSV) A and $\mathrm{B}^{15}$ adenoviruses, ${ }^{16}$ and rhinoviruses and enteroviruses. ${ }^{17}$ The PCR panel was used to identify L pneumophila, ${ }^{18} \mathrm{M}$ pneumoniae, ${ }^{10} \mathrm{C}$ pneumoniae, ${ }^{19}$ and $S$ pneumoniae using a novel PCR. This protocol was designed and optimised to target the pneumolysin gene using TGTTGAGACTAAGGTTACAGCT (PNLl) and ACCTGAgGatAgAgAgTtGTTC (PNL2) primers. Positive and negative controls were included in each series of up to 20 samples.

\section{Statistical analysis}

Statistical significance was assessed using the $\chi^{2}$ test for categorical variables and the Mann-Whitney $U$ test and Student's $t$ test for non-parametric and parametric continuous variables respectively using GB-STAT statistics software (Dynamic Microsystems, Inc, Silver Spring, USA). A p value of $<0.05$ was considered to be statistically significant. Data are presented as mean and standard deviations unless otherwise stated.

\section{RESULTS}

A total of 80 patients with acute LRTI and 49 controls were recruited during the 12 month study period. Recruited LRTI patients were representative of the adult LRTI population (table 1) as both groups were of similar age with a similar percentage of women, re-consultation rates, and GP antibiotic prescription rates. Smoking history and a history of obstructive lung disease were less common in the total LRTI population than in the recruited LRTI patients. There were no significant differences in age, percentage of women, and smoking histories between patients and controls $(\mathrm{p}>0.05)$, but a history of obstructive lung disease was less common in control subjects $(p=0.02)$. Throat swabs were collected from all patients and controls and nasal aspirates from 77 (96\%) and $48(98 \%)$, respectively. Sputum was collected from 60 patients $(75 \%)$. No patient entered the study on a second occasion.

\section{Clinical features of recruited LRTI patients and controls}

There were no significant differences between recruited LRTI patients and controls in temperature $(p=0.01)$, but respiratory rates, pulse rates, and CRP levels were significantly higher in patients $(\mathrm{p}<0.001$, table 2$)$. At presentation, the mean (SD) duration of the illness in patients was 8.7 (5.5) days. Symptoms were recorded in decreasing frequency as follows: cough $(100 \%)$, discoloured sputum $(83 \%)$, upper respiratory tract symptoms (81\%), breathlessness $(77 \%)$, wheeze $(71 \%)$, sweating (69\%), headache $(69 \%)$, fever $(60 \%)$, chest pain $(56 \%)$, myalgia $(50 \%)$, and haemoptysis $(9 \%)$.

\section{Antibiotic prescription for LRTI}

Antibiotics were prescribed to $64 \%$ of the recruited patients with LRTI (table 1). A second course of antibiotics was prescribed to $40 \%$ of the patients who re-consulted during the 4 weeks before the second study visit. 
Table 1 Demographic details of the general LRTI population, LRTI patients, and controls

\begin{tabular}{lllll}
\hline & $\begin{array}{l}\text { Total LRTI } \\
\text { population } \\
(\mathbf{n = 3 6 8 )}\end{array}$ & $\begin{array}{l}\text { Recruited LRTI } \\
\text { patients } \\
(\mathbf{n = 8 0 )}\end{array}$ & $\begin{array}{l}\text { Controls } \\
(\mathbf{n}=\mathbf{4 9})\end{array}$ & $\begin{array}{l}\text { p value (recruited } \\
\text { LRTI versus controls) }\end{array}$ \\
\hline Women (\%) & 63 & 63.8 & 63.3 & 0.894 \\
Mean (SD) age (years) & $52.1(19.8)$ & $49.9(19.7)$ & $49.7(17.3)$ & 0.91 \\
Age range & $18-97$ & $18-90$ & $22-83$ & NA \\
Smokers & $30 \%$ & $53.2 \%$ & $46.9 \%$ & 0.516 \\
History of asthma/COPD & $21 \%$ & $44.9 \%$ & $22.4 \%$ & 0.0178 \\
Antibiotic prescription & $64 \%$ & $64 \%$ & $\mathrm{NA}$ & $\mathrm{NA}$ \\
Re-consultation rate & $30 \%$ & $33 \%$ & $\mathrm{NA}$ & $\mathrm{NA}$ \\
\hline NA, not applicable. & & & & \\
\hline
\end{tabular}

\section{Aetiology of acute adult community acquired LRTI}

At least one organism was identified in 55 patients with LRTI compared with seven controls $(69 \% v 14 \% ; \mathrm{p}<0.0001)$. A total of 79 organisms ( 57 viruses, 22 bacteria) were identified in LRTI patients compared with nine (six viruses, three bacteria) in controls $(p<0.0001$, table 3$)$. The overall identification rates for the patient population were $63 \%$ for viruses and $26 \%$ for bacteria, compared with $12 \%(\mathrm{p}<0.0001)$ and $6 \%(p=0.013)$, respectively, for the control population, demonstrating the underlying carriage rates for these groups of organisms.

The most common viruses identified in patients with LRTI were rhinoviruses (in 33\%) and influenza viruses (in 24\%) compared with $2 \%(\mathrm{p}<0.001)$ and $6 \%(\mathrm{p}=0.013)$, respectively, in controls. The pneumolysin gene PCR detected $S$ pneumoniae in 15 LRTI patients (including the one patient who was $S$ pneumoniae sputum culture positive) and two controls $(\mathrm{p}=0.03)$. Sputum bacterial culture detected seven organisms (five $H$ influenzae, one $M$ catarrhalis, and one $S$ pneumoniae).

Multiple organisms were identified in 18 of the 80 patients with LRTI $(22.5 \%)$ and two of the 49 controls $(4 \%, p=0.011)$. Influenza viruses were the most common virus type associated with mixed viral and bacterial infection (10/15) followed by rhinoviruses (8/15). Mixed viruses were detected in eight patients, most commonly rhinovirus and influenza virus (7/8). Mixed bacterial infection was found in one patient with an associated mixed viral infection (H influenzae, $S$ pneumoniae, coronavirus and rhinovirus). In four patients three organisms were identified (three with $S$ pneumoniae, influenza virus and rhinovirus, one with $M$ pneumoniae, influenza virus and rhinovirus). Only six of 21 LRTI patients with bacteria detected had isolated bacterial infection (three $S$ pneumoniae, two $H$ influenzae, and one $M$ catarrhalis).

\section{DISCUSSION}

In this 12 month observational study we used nucleic acid amplification assays to detect both bacteria and viruses in acute adult LRTI in primary care. The majority of mild LRTI had a viral aetiology although mixed viral/bacterial infections were also common, and patients with a bacterial aetiology alone were not clinically distinguishable from those with viral LRTI. A low rate of recruitment led to a small study size; this was limited by the requirement for patients to meet with a second physician (the study Clinical Fellow) after the initial consultation with their GP. However, the study population was representative of the LRTI population presenting to the recruiting GPs. In particular, there were no significant differences in age, the proportion of women, prescription rate, or re-consultation rate observed (table 1). The characteristics of the patients in this study are also similar to recent studies performed in the UK which have reported a higher proportion of women and high prescription and reconsultation rates. ${ }^{3}$ Lower rates of smoking and obstructive lung disease in the entire LRTI population compared with the study population were noted and may be the result of underreporting of smoking and obstructive lung disease in the GP records. The incidence of LRTI of 37 per 1000 adult population per year in this study is also comparable to the rate of 44 per 1000 recently reported by Macfarlane et al, ${ }^{3}$ with the slightly lower rate coinciding with the reported low consultation levels for acute bronchitis and acute respiratory illness for England during the 2000/1 season. ${ }^{20}$

In this study an organism was detected in $69 \%$ of adults with acute moderate LRTI and was achieved by the use of PCR assays for the most common respiratory viruses and bacteria, and also by collection of specimens from throat swabs, nasal aspirates, and sputum. At the time of the study PCR assays were not available for $H$ influenzae or $M$ catarrhalis in our laboratory and addition of these may have further improved the rate of detection of pathogens. Even so, this detection rate is considerably higher than previous studies $\left(16 \%{ }^{8}\right.$ and $\left.55 \%{ }^{4}\right)$. In the present study respiratory viruses were the most common cause of acute adult LRTI, occurring in $63 \%$ of patients, while bacteria were detected in $26 \%$.

Previous studies have reported rates of virus detection ranging from $9 \%$ to $31 \%$ in acute adult LRTI using standard methods. ${ }^{4-8}$ The studies with higher detection rates relied heavily on serology for virus detection and these data could be interpreted as suggesting that viruses are not important

Table 2 Clinical features of patients with LRTI and controls

\begin{tabular}{|c|c|c|c|c|}
\hline & $\begin{array}{l}\text { LRTI }^{*} \\
(n=80)\end{array}$ & $\begin{array}{l}\text { Controls* } \\
(\mathrm{n}=49)\end{array}$ & $\begin{array}{l}\text { Mean difference } \\
\text { (upper/lower } \mathrm{Cl} \text { ) }\end{array}$ & $p$ value \\
\hline Respiratory rate & $20(4.99)$ & $14.7(3.32)$ & $\begin{array}{l}-5.24 \\
-6.84 \text { to }-3.65\end{array}$ & $<0.0001$ \\
\hline Pulse rate & $81.1(11.6)$ & $73.1(11.2)$ & $\begin{array}{l}-8.09 \\
-12.21 \text { to }-3.97\end{array}$ & $<0.001$ \\
\hline Temperature $\left({ }^{\circ} \mathrm{C}\right)$ & $36.4(0.52)$ & $36.2(0.47)$ & $\begin{array}{l}-0.23 \\
-0.41 \text { to }-0.05\end{array}$ & 0.01 \\
\hline CRP & $19.3(27.8)$ & $3.2(2.8)$ & $\begin{array}{l}-16.1 \\
-24.63 \text { to }-7.55\end{array}$ & $<0.001$ \\
\hline
\end{tabular}

$\mathrm{CRP}, \mathrm{C}$-reactive protein.

*Values shown as mean (SD) 


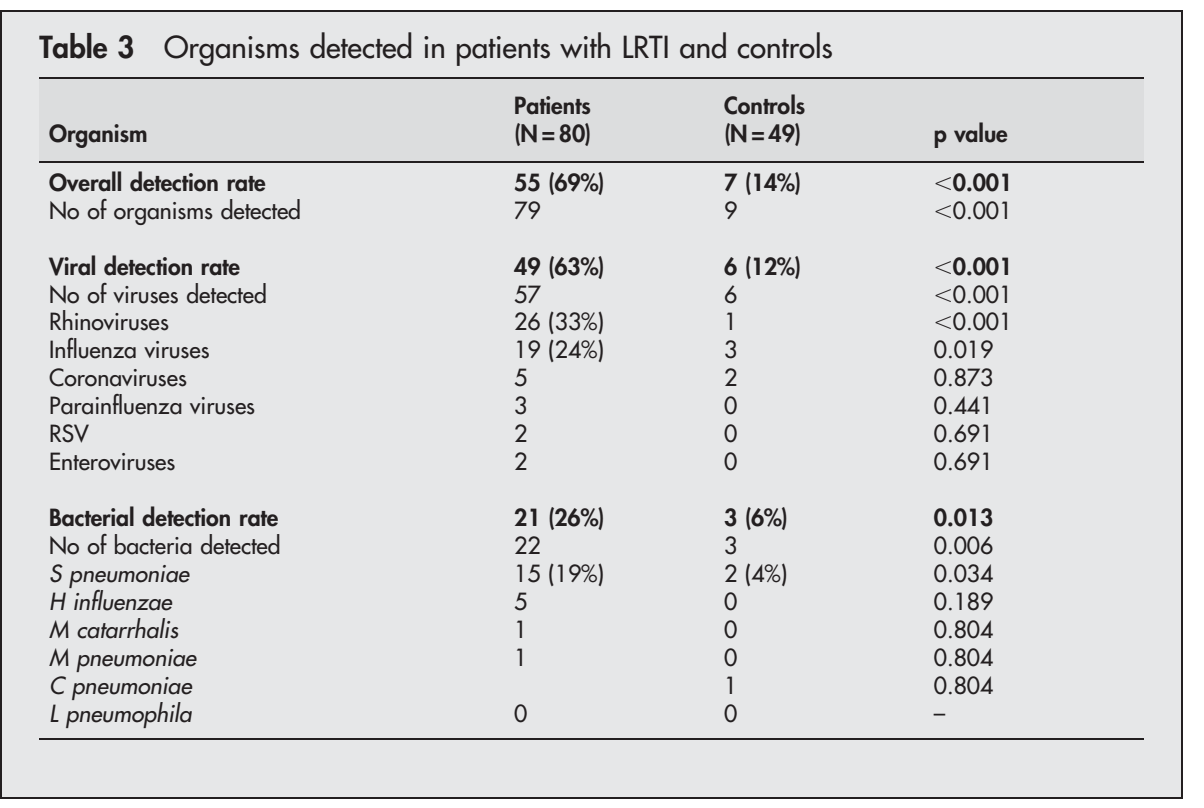

causes of acute adult LRTI. However, serological detection of rhinoviruses and coronaviruses is not widely used and viral culture is very insensitive. In this study, viruses were detected in $63 \%$ of patients using combined nasal aspiration and sputum sampling where available. The most common viruses were rhinoviruses (33\% of patients) followed by influenza viruses $(24 \%)$ and coronaviruses $(6 \%)$. A previous study by Macfarlane et $a l^{3}$ which used throat swabs alone achieved a total virus detection rate of $19 \%$, the three most common viruses being influenza, coronaviruses and rhinoviruses. Both the current study and the study by Macfarlane et al ${ }^{3}$ used PCR assays for virus detection, which suggests that the differences in viral detection rates may be due to the addition of nasal aspirates and sputum for virus PCR in our study. This is supported by previous studies in which nasal aspirates and sputum were used successfully with detection rates of 76$85 \% .^{21} 22$

Bacteria were detected in $26 \%$ of patients and the distribution of organisms corroborates data from previous studies in which bacterial detection varied between $6 \%$ and $26 \%,{ }^{367}$ indicating that direct detection of bacterial infection in acute adult LRTI in primary care is relatively uncommon. A bacterial yield of $44 \%$ was obtained in a primary care study of patients with LRTI treated with antibiotics using a combination of sputum culture (induced if necessary) and serology; $H$ influenzae (8\%) was cultured from sputum more commonly than $S$ pneumoniae $(6 \%)$ as in our study. However, the majority of bacterial infections, and particularly $S$ pneumoniae $(30 \%)$, were identified by serology and not by direct detection. ${ }^{4}$ The present study directly detected only a single case of $M$ pneumoniae. In comparison, the study by MacFarlane et $a l^{3}$-which reported the highest yield of infectious agents in adult LRTI in primary care to date (55\%)-reported that infection with atypical pathogens (predominantly $C$ pneumoniae) was inferred by serology in $25 \%$ of patients. PCR did not yield any positive samples in the study by MacFarlane et al. This difference may be due to the fact that recruitment occurred over different seasons, or the discrepancy between the serological and PCR results in the study by MacFarlane et al might result from use of throat swabs for the detection of these organisms by PCR. In our study, PCR in throat swabs, nasal aspirates, and sputum were used with concordant results and M pneumoniae was detected in a single patient. Other organisms were identified, which suggests that there were no significant inhibitors of PCR present in the assays. These data suggest that, in contrast to the study by Macfarlane et al using serology, ${ }^{3}$ M pneumoniae and $C$ pneumoniae were an uncommon cause of acute LRTI in adults in the season studied.

Co-infection with more than one organism was found in 18 of the 80 patients with LRTI $(22.5 \%)$, and the majority of these were mixed bacteria/viral infections (15/18, 83\%). Coinfection rates of $25 \%$ and $32 \%$ have been reported in previous LRTI studies. ${ }^{3}$ The clinical features and outcome of those with isolated bacterial infection were not significantly different from those with isolated viral infection. There is therefore no clinically useful way to differentiate between patients with bacterial infection and those with viral infection, and thus no clinical way to guide antibiotic treatment. Nevertheless, the prescription rate in the present study was $64 \%$, the same as the prescription rate for adult LRTI patients not recruited into the study and similar to the rate recently reported elsewhere for LRTI in primary care. ${ }^{3}$

This is the first study of LRTI in primary care to compare patients with a matched control population, permitting aetiological relationships to be inferred. This is particularly important in studies using sensitive methods of detection such as PCR, and inclusion of controls would have aided interpretation of previous studies that relied heavily on serology for diagnosis. In the present study controls were well matched for age, percentage of women, and smoking history. A history of obstructive lung disease was significantly more common in recruited LRTI patients than in controls. The significantly higher respiratory rate and CRP levels in patients compared with controls suggest the likelihood of patients having an infective respiratory illness $(\mathrm{p}<0.001)$. The significantly higher identification rates in the patient population $(69 \%)$ than in controls $(14 \%, p<0.001)$ and the similarity of our detection rate in matched controls to previous reports ${ }^{23}$ provide confirmation of the causative role of the majority of the pathogens detected in this study.

In summary, we have shown that LRTI in primary care is predominantly a viral illness with a high rate of antibiotic prescription that is unrelated to bacterial aetiology. Infection with atypical bacterial pathogens was negligible.

\section{ACKNOWLEDGEMENTS}

The authors thank the general practitioners and nurse practitioners who helped with recruitment and specimen collection at both the Hampstead group practice and Lawrence House surgeries; Dr Richard Morris, Royal Free and University College Medical School, for help 
with the statistics; and Mike Edwards for help with the picornavirus digests. The laboratory work could not have been achieved without the meticulous sample processing and archiving by staff members of the Department of Medical Microbiology, Royal Free Hospital.

\section{Authors' affiliations}

D D Creer, Barnet General Hospital, Wellhouse Lane, Barnet EN5 3DJ, UK

J P Dilworth, A R Johnston, Department of Thoracic Medicine, Royal Free and University College Medical School, University College London, UK

S H Gillespie, C Ling, S Patel, T D McHugh, Centre for Medical Microbiology, Royal Free and University College Medical School, University College London, UK

S L Johnston, G Sanderson, Department of Respiratory Medicine, National Heart and Lung Institute \& Wright Fleming Institute of Infection and Immunity, Imperial College, London, UK

P G Wallace, Department of Primary Care and Population Sciences, Royal Free and University College Medical School, University College London, UK

The study received funding and support from the North Central Thames Primary Care Research Network (NoCTeN) and Royal Free NHS Trust Collaborative Project Grant. GS was supported by a British Lung Foundation/Severin Wunderman Family Foundation Lung Research Programme Grant awarded to SL.

Competing interests: none.

\section{REFERENCES}

1 Office of Population Censuses and Surveys. Morbidity statistics from general practice, Fourth national study, 1991-1992. Series MB5,3. London: HMSO, 1995.

2 Huchon G, Woodhead M, Gialdroni-Grassi G, et al. Management of adult community-acquired lower respiratory tract infections. Eur Respir Rev 1998;8:389-426

3 Macfarlane J, Holmes W, Gard P, et al. Prospective study of the incidence, aetiology and outcome of adult lower respiratory tract illness in the community. Thorax 2001;56:109-14.

4 Macfarlane JT, Colville A, Guion A, et al. Prospective study of aetiology and outcome of adult lower-respiratory-tract infections in the community. Lancet 1993;341:511-4.

5 Standing Medical Advisory Committee Subgroup on Antimicrobial Resistance. The path of least resistance. London: Department of Health, 1998.

6 Boldy DAR, Skidmore SJ, Ayres JG. Acute bronchitis in the community: clinical features, infective factors, changes in pulmonary function and bronchial reactivity to histamine. Respir Med 1990;84:377-85.
7 Melbye H, Berdal BP. Akutt bronkitt hos voksne. Kliniske funn, mikroorganismer og bruk av antibiotika [Acute bronchitis in adults. Clinical findings, microbes and antibacterial treatment]. Tidsskr Nor Laegeforen 1994;114:814-7.

8 Jonsson JS, Sigurdsson JA, Kristinsson KG, et al. Acute bronchitis in adults. How close do we come to its aetiology in general practice? Scand J Primary Health Care 1997; 15:156-60.

9 Johnston SL, Sanderson G, Pattemore PK, et al. Use of polymerase chain reaction for diagnosis of picornavirus infection in subjects with and without respiratory symptoms. J Clin Microbiol 1993;31:111-7.

10 leven M, Ursi D, Van Bever H, et al. Detection of Mycoplasma pneumoniae by two polymerase chain reactions and role of $M$ pneumoniae in acute respiratory tract infections in pediatric patients. J Infect Dis 1996;173:1445-52.

11 Cunningham AF, Johnston SL, Julious SA, et al. Chronic Chlamydia pneumoniae infection and asthma exacerbations in children. Eur Respir $J$ 1998; 11:345-9.

12 Ellis JS, Fleming DM, Zambon MC. Multiplex reverse transcription-PCR for surveillance of influenza A and B viruses in England and Wales in 1995 and 1996. J Clin Microbiol 1997;35:2076-82.

13 Corne JM, Green S, Sanderson G, et al. A multiplex RT-PCR for the detection of parainfluenza viruses 1-3 in clinical samples. J Virol Methods 1999:82:9-18.

14 Myint S, Johnston S, Sanderson G, et al. Evaluation of nested polymerase chain methods for the detection of human coronaviruses $229 \mathrm{E}$ and OC43. Mol Cell Probes 1994;8:357-64.

15 O'Donnell DR, McGarvey MJ, Tully JM, et al. Respiratory syncytial virus RNA in cells from the peripheral blood during acute infection. J Pediatr 1998; 133:272-4

16 Hierholzer JC, Halonen PE, Dahlen PO, et al. Detection of adenovirus in clinical specimens by polymerase chain reaction and liquid-phase hybridisation quantitated by time-resolved fluorometry. J Clin Microbiol 1993;31:1886-91.

17 Papadopoulos NG, Hunter J, Sanderson G, et al. Rhinovirus identification by Bgll digestion of picornavirus RT-PCR amplicons. J Virol Methods 1999;80:179-85.

18 Jaulhac B, Nowicki M, Bornstein N, et al. Detection of Legionella spp in bronchoalveolar lavage fluids by DNA amplification. J Clin Microbiol 1992;30:920-4.

19 Campbell LA, Melgosa MP, Hamilton DJ, et al. Detection of Chlamydia pneumoniae by polymerase chain reaction. J Clin Microbiol 1992;30:434-9.

20 Goddard N. Summary of activity for 2000/01 season, PHLS Weekly Influenza Report UK Surveillance of influenza and other seasonal respiratory illness. London: PHLS, 2001

21 Johnston SL, Pattemore PK, Sanderson G, et al. Community study of role of viral infections in exacerbations of asthma in 9-11 year old children. BMJ 1995;310:1225-9.

22 Wark PA, Johnston SL, Moric I, et al. Neutrophil degranulation and cell lysis is associated with clinical severity in virus-induced asthma. Eur Respir $J$ 2002; 19:68-75.

23 Fransen H, Wolontis S. Infections with viruses, Mycoplasma pneumoniae and bacteria in acute respiratory illness. A study of hospitalized patients, patients treated at home, and healthy subjects. Scand J Infect Dis 1969;1:31-7. 